\title{
Rate of abnormal vaginal bleeding and contraception counseling in women undergoing chemotherapy
}

\author{
Kristin E Cutler, BS, ${ }^{a}$ Mitchell D Creinin, MD, Jay Michael S Balagtas, MD, ${ }^{\mathrm{b}}$ and Melody \\ Y Hou, MD, $\mathrm{MPH}^{\mathrm{a}}$
}

${ }^{a}$ Department of Obstetrics and Gynecology, University of California, Davis, Sacramento, California; and ${ }^{\mathrm{b}}$ Division of Pediatric Hematology-Oncology, Stanford University, California

Background Reproductive-age women who are undergoing cancer treatment are at risk for heavy menstrual bleeding, unintended pregnancy, and have a contraindication to estrogen-containing products. The incidence of vaginal bleeding and contraception use is not known.

Objective To describe clinical practices regarding menstrual suppression, estimate the incidence of vaginal bleeding complaints, and investigate contraceptive counseling and provision in women undergoing chemotherapy.

Methods We performed a chart review using ICD-9 codes to identify women aged 14-40 years who received chemotherapy at our institution during July 2008-June 2013. Electronic medical records were examined for menstrual suppression therapy, contraception counseling, and abnormal vaginal bleeding.

Results We identified 137 women for this study. 24 (18\%) received prophylactic menstrual suppression counseling, of whom 17 $(71 \%)$ initiated treatment, primarily with combined oral contraceptives, all prescribed by hematologist-oncologists. During the first 6 months of chemotherapy, 36 women (26\%) complained of abnormal vaginal bleeding, including 10 women who were on prophylactic treatment. 19 women noted moderate to severe bleeding. 11 (12\%) women received contraceptive counseling before chemotherapy, all from hematologist-oncologists; 86 women (63\%) initiated chemotherapy without a documented contraceptive method.

Limitations Data were generated from billing codes, so all eligible women may not have been included.

Conclusions Reproductive-age women undergoing chemotherapy may not receive adequate contraception and menstrual suppression counseling. We encourage consulting gynecologists, especially family planning specialists, at the time of cancer diagnosis to support women with decisions about menstrual suppression and contraception.

Funding/sponsorship Society of Family Planning, National Center for Advancing Translational Sciences, National Institutes of Health, Lillian Mae Rapp Research Endowment

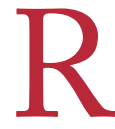

eproductive-age women who undergo treatment for oncologic disease have a risk of up to $40 \%$ of developing heavy menstrual bleeding as a direct effect of hematologic malignancy or secondary to myelosuppressive chemotherapy or bone marrow transplant (BMT)., ${ }^{1,2}$ Commonly recommended menstrual suppression agents include estrogen-containing contraceptives, progestin-only methods, and gonadotropin-releasing $(\mathrm{GnRH})$ agonists for vaginal bleeding complaints related to heavy or irregular bleeding in chemotherapy patients, but these practices rely on limited data. ${ }^{2-5}$

Some experts specifically recommend the use of continuous combined estrogen and progesterone contraceptives to suppress menstruation in women undergoing BMT. ${ }^{3}$ However, the Centers for Disease
Control and Prevention (CDC) and the Society of Family Planning advise that estrogen-containing contraceptives should be avoided in women with active cancer because of the increased risk of venous thromboembolism (VTE). ${ }^{6,7}$ Reproductive-age women with cancer also suffer from increased risk of unintended pregnancy after treatment. ${ }^{8,9}$ The rate of contraception counseling and use in this population is not known.

This report describes the clinical practices regarding menstrual suppression in women treated for oncologic disease, including counseling and therapies at our institution. We also aimed to estimate the incidence of vaginal bleeding complaints related to heavy or irregular bleeding and to describe the contraceptive counseling and provision among our patients.

Accepted for publication August 4, 2016. Correspondence: Melody Y Hou, MD, MPH; myhou@ucdavis.edu. Disclosures: The authors report no disclosures or conflicts of interest. JCSO 2016;14(8):337-341. @2016 Frontline Medical Communications. doi: 10.12788/icso.0290. 
TABLE 1 Demographics of women receiving chemotherapy for hematologic cancers and malignant solid tumors (July 1, 2008 to June 31, 2013; $N=137$ ).

\begin{tabular}{|c|c|}
\hline Demographic & No. of patients (\%) \\
\hline Mean age: 29 y $(S D, 8)$ & - \\
\hline \multicolumn{2}{|l|}{ Race } \\
\hline $\begin{array}{l}\text { Latina } \\
\text { White } \\
\text { Black } \\
\text { Asian/Pacific Islander } \\
\text { Other } \\
\text { Not recorded }\end{array}$ & $\begin{array}{c}33(24) \\
32(23) \\
6(4) \\
13(9) \\
1(1) \\
52(38)\end{array}$ \\
\hline \multicolumn{2}{|l|}{ Gravidity } \\
\hline $\begin{array}{l}0 \\
1 \\
\geq 2 \\
\text { Not recorded }\end{array}$ & $\begin{array}{c}39(28) \\
11(8) \\
32(23) \\
55(40)\end{array}$ \\
\hline \multicolumn{2}{|l|}{ Parity } \\
\hline $\begin{array}{l}0 \\
1 \\
\geq 2 \\
\text { Not recorded }\end{array}$ & $\begin{array}{l}42(31) \\
27(20) \\
39(28) \\
29(21)\end{array}$ \\
\hline \multicolumn{2}{|l|}{ Insurance status } \\
\hline $\begin{array}{l}\text { Self-pay } \\
\text { Private } \\
\text { Medicaid }\end{array}$ & $\begin{array}{c}8(6) \\
67(49) \\
62(45)\end{array}$ \\
\hline \multicolumn{2}{|l|}{ Contraception ${ }^{a}$} \\
\hline $\begin{array}{l}\text { None recorded } \\
\text { Abstinence } \\
\text { CHC } \\
\text { Sterilization } \\
\text { Hormonal IUD } \\
\text { Copper IUD } \\
\text { Condoms } \\
\text { Fertility awareness }\end{array}$ & $\begin{array}{l}89(65) \\
14(10) \\
9(7) \\
8(6) \\
8(6) \\
4(3) \\
4(3) \\
1(1)\end{array}$ \\
\hline \multicolumn{2}{|c|}{$\begin{array}{l}\mathrm{CHC} \text {, combined hormonal contraception (pills, vaginal ring, patch); IUD, } \\
\text { intrauterine device }\end{array}$} \\
\hline Before chemotherapy. & \\
\hline
\end{tabular}

\section{Methods}

We performed a retrospective chart review of women aged 14-40 years who received chemotherapy for hematologic cancers and malignant solid tumors at the University of California, Davis Medical Center, Sacramento, during July 1, 2008-June 31, 2013. Using ICD-9 diagnosis codes for leukemia (200-202), lymphoma (204-208), and malignant solid tumors (140-199) as recorded in the electronic medical record, we identified all women who initiated chemotherapy at our institution during the study period. Exclusion criteria included initiation of chemotherapy outside of the center, premenarchal or menopausal status, having had a hysterectomy or bilateral oophorectomy before chemotherapy, pregnancy during chemotherapy, or tumor of gynecologic etiology. The University of California
Davis institutional review board approved this study.

We primarily investigated the rate of menstrual suppression counseling before chemotherapy initiation. The secondary outcome was a complaint of vaginal bleeding within the first 6 months of chemotherapy. We defined a vaginal bleeding complaint as any concern about heavy or irregular vaginal bleeding within 6 months of chemotherapy initiation expressed by the patient or clinician and documented in the medical record. We extracted these data, along with demographic data, chemotherapy duration, cancer diagnosis, chemotherapy agents received, hematologic data before and after chemotherapy and during bleeding episodes, timing and use of menstrual suppression therapies, and details regarding contraceptive counseling and initiation, from the electronic medical records into Research Electronic Data Capture (REDCap). We used hematologic data within 1 month of the date of interest for analyses. For example, a hemoglobin level at 6 months after chemotherapy initiation would be from a sample drawn between days 150 and 210 following chemotherapy initiation, with a preference for the sample drawn closest to day 180 . We limited our data collection to medical records only and made no attempts to contact patients or clinicians.

We analyzed continuous variables such as age by 2 -sample $t$ test or 1-way ANOVA, unless a non-normal distribution called for a Wilcoxon rank sum test, categorical variables by chi-square tests, and proportions with the Fisher exact test using SAS 9.3 (Cary, NC).

\section{Results}

During the 5-year period (July 2008-June 2013), 1,468 women aged 14-40 years, inclusive, had an office visit associated with leukemia, lymphoma, or malignant solid tumor. All of their charts were available for review, and 137 patients met criteria for analysis. The mean age of our study population was 29 (SD, 8) years. Patients were primarily nulliparous, racially and ethnically diverse, and insured (Table 1).

Twenty-four women (18\%, 95\% confidence interval [CI], 12\%-24\%) received menstrual suppression counseling before the start of chemotherapy to prophylactically address heavy menstrual bleeding as a complication of thrombocytopenia ( $\mathrm{n}=13)$, fertility preservation $(\mathrm{n}=7)$, ovarian suppression as a treatment modality for breast cancer $(n=3)$, and for no specified reason $(n=1)$. Hematologist-oncologists performed most of this counseling (22 hematologist-oncologists, 2 gynecologists). Seventeen women (71\%) elected to start prophylactic menstrual suppression and received combined oral contraception (COC; $\mathrm{n}=13$ ), $\mathrm{GnRH}$ agonists ( $\mathrm{n}$ $=2)$, or depot medroxyprogesterone acetate $(n=2)$. All of these methods were prescribed by hematologist-oncologists.

Among the 137 women in our study population, 36 (26\%, 95\% CI, 19\%-33\%) complained of abnormal vaginal bleeding during the first 6 months of chemotherapy, with 19 (14\%) experiencing moderate to severe bleeding. Women 


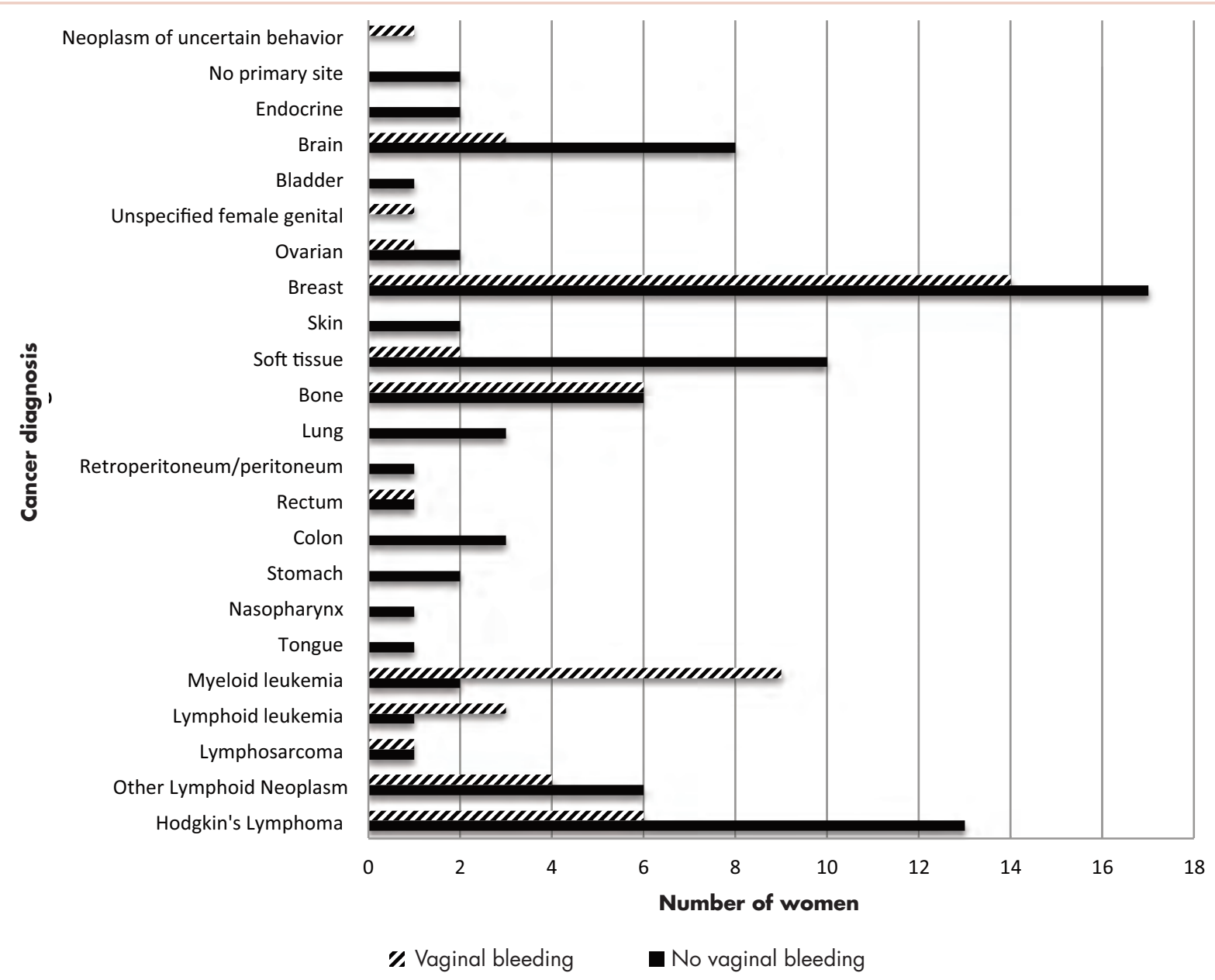

FIGURE 1 Number of women experiencing abnormal vaginal bleeding within the first 6 months of chemotherapy, categorized by ICD-9 cancer diagnosis $(\mathrm{N}=137)$.

with myeloid leukemia had the highest rate of vaginal bleeding, affecting 9 of 11 patients with this diagnosis (Figure). Complaints of abnormal bleeding were more frequent among women who received prophylactic menstrual suppression than among those who did not (9 of 17 [53\%] vs 27 of 120 [23\%], respectively, $P=.02)$. Women who received radiation in addition to their chemotherapy did not have a significantly higher rate of abnormal vaginal bleeding compared with women who received chemotherapy alone (17 of $51(33 \%)$ vs 19 of $86(22 \%)$, respectively, $P=.16)$.

Nineteen of 36 women (53\%) who experienced abnormal vaginal bleeding received counseling for therapeutic menstrual suppression. As noted with prophylactic menstrual suppression, COCs were the most commonly prescribed therapeutic agent (13 of 19,68\%) and all were prescribed by hematologist-oncologists.

We compared platelet counts and hemoglobin levels that had been evaluated before chemotherapy initiation, 6 months after chemotherapy initiation, and at the time of the first bleeding episode in women with abnormal bleeding. Only 107 of the 137 women in the study had hemoglobin and platelet counts drawn at 6 months after chemotherapy initiation. Median hemoglobin levels did not significantly differ at baseline or at 6 months after chemotherapy among women who later experienced abnormal vaginal bleeding compared with those who did not have abnormal bleeding (Table 2). Platelet levels were significantly lower at 6 months after chemotherapy compared with baseline in both groups, but remained within normal limits (higher than 150 cells $\mathrm{x}$ $10^{3} / \mathrm{uL}$ ). At the time of their first bleeding episode, women with abnormal bleeding had median platelet levels significantly lower than baseline $\left(119 \times 10^{3} / \mathrm{uL}, P=.03\right)$.

When women were categorized by level of thrombocytopenia before chemotherapy, 6 months after treatment, and during a first bleeding episode (Table 3), severe thrombocytopenia was more likely to be present during a reported bleeding episode than at 6 months in women who never experienced abnormal bleeding (29\% vs 3\% respectively; 
TABLE 2 Median hemoglobin and platelet counts ${ }^{a}$ in patients who did and did not experience abnormal vaginal bleeding during chemotherapy $(\mathrm{N}=107)^{\mathrm{b}}$

\begin{tabular}{|c|c|c|c|c|c|c|}
\hline & \multicolumn{2}{|c|}{ Before chemotherapy } & \multicolumn{2}{|c|}{$\begin{array}{c}6 \text { mo. after } \\
\text { chemotherapy initiation }\end{array}$} & \multicolumn{2}{|c|}{ First bleeding episode } \\
\hline & $\begin{array}{l}\text { Hemoglobin, } \\
\text { g/dL (range) }\end{array}$ & $\begin{array}{l}\text { Platelets, } 10^{3} / \\
\text { uL (range) }\end{array}$ & $\begin{array}{l}\text { Hemoglobin, } \\
\text { g/dL (range) }\end{array}$ & $\begin{array}{l}\text { Platelets, } \\
10^{3} / \text { uL (range) }\end{array}$ & $\begin{array}{l}\text { Hemoglobin, } \\
\text { g/dL (range) }\end{array}$ & $\begin{array}{l}\text { Platelets, } \\
10^{3} / \text { uL (range) }\end{array}$ \\
\hline $\begin{array}{l}\text { With abnormal vaginal } \\
\text { bleeding }(n=35)\end{array}$ & $\begin{array}{c}10.5 \\
(7-13.7)\end{array}$ & $\begin{array}{c}261 \\
(29-595)\end{array}$ & $\begin{array}{c}11.2 \\
(7.4-15.3)\end{array}$ & $\begin{array}{c}196^{\text {cd }} \\
(8-365)\end{array}$ & $\begin{array}{c}9.8 \\
(7-15.4)\end{array}$ & $\begin{array}{c}119^{e} \\
(7-312)\end{array}$ \\
\hline $\begin{array}{l}\text { Without abnormal vaginal } \\
\text { bleeding }(n=72)\end{array}$ & $\begin{array}{c}11.4 \\
(7.5-14.5)\end{array}$ & $\begin{array}{c}292 \\
(13-794)\end{array}$ & $\begin{array}{c}11.1 \\
(7.3-14.5)\end{array}$ & $\begin{array}{c}240^{f} \\
(4-626)\end{array}$ & - & - \\
\hline
\end{tabular}

$P=.0002)$. However, most of the women with abnormal bleeding still had a normal platelet count of $>150 \times 10^{3} / \mathrm{uL}$.

Most women ( $n=89,65 \%)$ did not have documentation of pregnancy risk or contraceptive status in their medical records before chemotherapy initiation. Only 11 of those women received contraceptive counseling before chemotherapy initiation, provided in all cases by hematologist-oncologists. After counseling, 1 patient chose to use condoms, 2 chose COCs, and 8 patients did not have a contraception plan for chemotherapy. Overall, 86 women (63\%; 95\% CI, 55\%-71\%) initiated chemotherapy without documentation of pregnancy risk or contraception use.

\section{Discussion}

More than a quarter of reproductive-age women who were undergoing chemotherapy at our institution experienced abnormal vaginal bleeding. Fewer than $20 \%$ of women received counseling about prophylactic menstrual suppression, only half were advised about treatment options after they had a bleeding complaint, and hematologist-oncologists performed nearly all of the gynecologic counseling. Estrogen-containing contraception was the most frequently prescribed method for bleeding prophylaxis, which is controversial given the already high risk of this population for thrombotic events. ${ }^{6,7}$ In contrast to the findings of Meirow and colleagues, ${ }^{1}$ women receiving prophylaxis for bleeding in our population had a higher rate of vaginal bleeding complaints related to heavy or irregular bleeding compared with those who did not. One explanation may be that our clinicians chose to prescribe prophylaxis in women in whom they had the highest suspicion for bleeding, although these prophylactic measures were ultimately unsuccessful. We did not find differing rates of vaginal bleeding between women who received chemoradiation versus chemotherapy alone, although our study population may have been too small to have detected a meaningful difference.

Although the median hemoglobin and platelet levels in women with abnormal vaginal bleeding decreased during

TABLE 3 Number of women experiencing various levels of thrombocytopenia before chemotherapy, during chemotherapy, and first abnormal vaginal bleeding episode during chemotherapy $(\mathrm{N}=107)^{\mathrm{a}}$

\section{Level of thrombocytopenia, n (\%)}

$\begin{array}{cccc}\text { Normal } & \text { Mild } & \text { Moderate } & \text { Severe } \\ \left(>150 \times 10^{3} / \mathrm{uL}\right) & \left(100-149 \times 10^{3} / \mathrm{uL}\right) & \left(50-99 \times 10^{3} / \mathrm{uL}\right) & \left(<50 \times 10^{3} / \mathrm{uL}\right)\end{array}$

With abnormal vaginal

bleeding $(\mathrm{n}=35)$

\begin{tabular}{|c|c|c|c|c|}
\hline Before chemotx & 27 (77) & $2(6)$ & $3(9)$ & $3(9)$ \\
\hline $\begin{array}{l}\text { During first bleeding } \\
\text { episode }\end{array}$ & $16(46)$ & $5(14)$ & $4(11)$ & $10(29)$ \\
\hline
\end{tabular}

\begin{tabular}{|c|c|c|c|c|}
\hline \multicolumn{5}{|c|}{$\begin{array}{l}\text { Without abnormal vaginal } \\
\text { bleeding }(n=72)\end{array}$} \\
\hline Before chemotx & $65(90)$ & $6(8)$ & 0 & $1(1)$ \\
\hline 6 mo after chemotx & $61(85)$ & $7(10)$ & $2(3)$ & $2(3)$ \\
\hline
\end{tabular}

chemotx, chemotherapy

anly 107 of the 137 women evaluated had 6-month hematologic data available for analysis. 
chemotherapy, most of those with vaginal bleeding complaints $(71 \%)$ had normal platelet levels. Therefore, platelet count alone may not be a reliable predictor of bleeding abnormalities among chemotherapy patients. We did not correlate hemoglobin or platelet levels with transfusion or report transfusion rates because we could not discern clearly in this retrospective review whether the reason for transfusion in this patient population was directly related to vaginal bleeding or anemia for other reasons.

Although most of the physicians counseled about menstrual suppression out of concern for possible thrombocytopenia-induced heavy menstrual bleeding, about a third of them documented fertility preservation as the primary reason to induce amenorrhea during treatment. Multiple societies and organizations have cautioned about the limited evidence about efficacy, the risks or benefits of ovarian suppression during chemotherapy, and that women should only do so in the setting of a clinical trial or after they have been referred to specialists with expertise in fertility preservation. ${ }^{10-12} \mathrm{We}$ also found that women infrequently received contraception counseling before chemotherapy initiation. Of those who are counseled, 63\% still began chemotherapy without contraception, which is a higher rate than the $38 \%$ of the general US population of women who are not using contraception. ${ }^{13}$

Limitations of the study include the unknown generalizability of a single institution's experience and its retrospective nature, which is affected not only by clinician bias but also by provider documentation and coding deficiencies that may miss eligible women. Another limitation is our lack of documentation about pregnancy risk. None of the hematologist-oncologists recorded sexual orientation or sexual activity; therefore, we may have overestimated the need for contraception counseling. We may also have underestimated the rate of vaginal bleeding if patients did not report abnormal bleeding episodes to their physicians. A prospective cohort study would provide more insight into women's gynecologic problems while on chemotherapy. However, this study describes real-world practices of hematologist-oncologists in an academic institution who treat women newly diagnosed with cancer and about to begin chemotherapy.

Receiving a new diagnosis of cancer is a vulnerable time for young women and has repercussions for multiple aspects of their lives, including current and future reproductive concerns. Hematologist-oncologists who provide counseling on all of these aspects may not have enough knowledge or experience to address possible gynecologic issues. For example, the most effective reversible contraceptive options, the levonorgestrel-releasing intrauterine device and the contraceptive implant, are progestin-only methods that can suppress menstruation without increased risk of VTE. These methods may not be familiar to many hematologist-oncologists. Concerns about infection associated with using intrauterine contraceptives for women undergoing chemotherapy should be addressed with further clinical trials, but can be otherwise mitigated by placement during non-neutropenic/leukopenic periods and judicious patient selection (based on oncologic disease type and the anticipated chemotherapy regimen). We should also note that the CDC has already recommended intrauterine contraceptives as reasonable and safe options for women with other immunosuppressive conditions based on the best available evidence. ${ }^{6,7,14}$ Obstetriciangynecologists, particularly family planning specialists, can offer a unique resource for women and their hematologistoncologists at the time of diagnosis. We encourage a crossdiscipline approach or at least consultation with obstetrics and gynecology at the time of diagnosis to assist women and their hematologist-oncologists with counseling about menstrual suppression, fertility preservation, and contraception.

\section{References}

1. Meirow D, Rabinovici J, Katz D, Or R, Shufaro Y, Ben-Yehuda D. Prevention of severe menorrhagia in oncology patients with treatment-induced thrombocytopenia by luteinizing hormone-releasing hormone agonist and depo-medroxyprogesterone acetate. Cancer. 2006;107:1634-1641.

2. Amsterdam A, Jakubowski A, Castro-Malaspina H, et al.Treatment of menorrhagia in women undergoing hematopoietic stem cell transplantation. Bone Marrow Transplant. 2004;34:363-366.

3. Adegite E, Goyal R, Murray P, Marshal M, Sucato, G. The management of menstrual suppression and uterine bleeding: a survey of current practices in the Pediatric Blood and Marrow Transplant Consortium. Pediatr Blood Cancer. 2012;59:553-557.

4. Chang K, Merideth M, Stratton P. Hormone use for therapeutic amenorrhea and contraception during hematopoietic cell transplantation. Obstet Gynecol. 2015;126:779-784.

5. Committee opinion no. 606: Options for prevention and management of heavy menstrual bleeding in adolescent patients undergoing cancer treatment. Obstet Gynecol. 2014;124:397-402.

6. Centers for Disease Control and Prevention. Update to CDC's US Medical Eligibility Criteria for Contraceptive Use, 2010: revised recommendations for the use of hormonal contraception among women at high risk for HIV infection or infected with HIV. MMWR Morb Mortal Wkly Rep. 2012;61:449-452.

7. Patel A, Schwarz E. Cancer and contraception. Release date May 2012. SFP Guideline \#20121. Contraception, 2012;86:191-198.

8. Green D, Whitton J, Stovall M, et al. Pregnancy outcome of female survivors of childhood cancer: a report from the Childhood Cancer Survivor Study. Am J Obstet Gynecol. 2002;187:1070-1080.

9. Murphy D, Klosky JL, Termuhlen A, Sawczyn KK, Quinn GP. The need for reproductive and sexual health discussions with adolescent and young adult cancer patients. Contraception. 2013;88:215-220.

10. Elgindy E, Sibai H, Abdelghani A, Mostafa, M. Protecting ovaries during chemotherapy through gonad suppression: a systematic review and meta-analysis. Obstet Gynecol. 2015;126:187-195.

11. Lee S, Schover L, Partridge A, et al. American Society of Clinical Oncology recommendations on fertility preservation in cancer patients. J Clin Oncol. 2006;24:2917-2931.

12. Loren A, Mangu P, Beck L, et al. Fertility preservation for patients with cancer: American Society of Clinical Oncology clinical practice guideline update. J Clin Oncol. 2013;31:2500-2510.

13. Jones J, Mosher W, Daniels K. Current contraceptive use in the United States, 2006-2010, and changes in patterns of use since 1995. Natl Health Stat Report. 2012;60:1-25.

14. Ramhendar T, Byrne P. Use of the levonorgestrel-releasing intrauterine system in renal transplant recipients: a retrospective case review. Contraception. 2012;86:288-289. 